\title{
Cohésion et énergie - la FMH en 2010
}

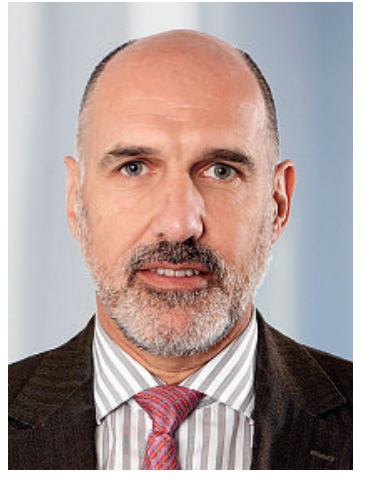

De tout ce qu'on peut en sentir, à l'interne et à l'externe, 2010 sera pour la FMH une année positive de consolidation et de nouveaux pas en avant. Cela demandera, évidemment, que la cohésion et l'énergie nécessaires soient là.

Cohésion, car les sujets ne manquent en réalité pas qui pourraient mettre sous tension les liens qui rassemblent le corps médical.

Les questions tarifaires, les projets de réseaux de soins, la propharmacie, l'initiative pour la médecine de premier recours, ou encore la mise en place de l'Institut pour la formation médicale (ISFM) ou les préparatifs en vue des DRG, sont autant de domaines dans lesquels nous devrons activement (activement!), tous et toutes, serrer les coudes pour exister dans les débats, et imposer nos idées.

Energie, ensuite, car rien n'est jamais acquis, bien sûr, et la position favorable que nous avons progressivement atteinte dans le registre politique doit être inlassablement consolidée, ce qui est la tâche commune, collective, de toutes celles et de tout ceux parmi nous qui s'engagent pour le corps médical.

\section{Les sujets ne manquent dans lesquels nous devrons activement serrer les coudes pour exister dans les débats}

De même, les canaux de communication maintenant ouverts entre partenaires du système de santé devront être entretenus, car on a là les meilleures chances de pouvoir développer dans les temps qui viennent un système de santé durable, au-delà des slogans à la mode et de certains activismes.

Cohésion et énergie ...

L'équilibre, équilibre entre les groupes constitutifs de la FMH comme entre les diverses instances statutaires qui nous structurent, l'équilibre des pouvoirs en fait, tient une place essentielle dans l'esprit et dans les projets du Comité central de la FMH.

\section{Les canaux de communication mainte-} nant ouverts entre partenaires du système de santé devront être entretenus

Car un corps médical qui ne s'unirait pas sur les options fondamentales qui le définissent et qui définissent son travail, manquera assurément de la force nécessaire pour définir sa place et pour la tenir, pour définir son rôle dans la société et pour le remplir.

Je vous exhorte donc vivement, chères Collègues, chers Collègues, à considérer tant notre métier que nos institutions professionnelles en fonction de l'avenir, en fonction des temps qui viennent, et à ne pas en faire les victimes malheureuses d'options, de visions caduques.

Nous médecins, nous FMH, avons notre place dans la société des temps qui viennent - prenons-la, cette place !

Je vous présente tous mes vœux pour une excellente année 2010.

Dr Jacques de Haller, Président de la FMH 